\title{
Comparison of Denoising Filters on Greyscale TEM Image for Different Noise
}

\author{
Garima Goyal ${ }^{1}$, Ajay Kumar Bansal ${ }^{2}$, Manish Singhal ${ }^{3}$ \\ ${ }^{1}$ Student-Mtech, ${ }^{2}$ Department of Electrical Engineering ${ }^{3}$ Department of Electronics Communication \& \\ Engineering \\ ${ }^{1,3}$ Poornima College of Engineering ${ }^{2}$ Poornima Institute of Engineering \& Technology
}

\begin{abstract}
TEM (Transmission Electron Microscopy) are currently the most widely used techniques to study nanoparticles morphology. Removal of noise from an image is one of the most important tasks in image processing. Depending on the nature of the noise, such as additive or multiplicative type of noise, there are several approaches towards removing noise from an image. Image De-noising improves the quality of images acquired by optical, electro-optical or electronic microscopy. This paper compares five filters on the measures of mean of image, signal to noise ratio, peak signal to noise ratio \& mean square error. In this work four types of noise (Gaussian noise, Salt \& Pepper noise, Speckle noise and Poisson noise) is used and image de-noising performed for different noise. Further results have been compared for all noises. . In this paper four types of noise are used and image de-noising performed for different noise by various filters (WFDWT, BF, HMDF, FDE, and DVROFT). Further results have been compared for all noises. It is observed that for Gaussian Noise WFDWT \& for other noises HMDF has shown the better performance results.
\end{abstract}

Keyword: Nonmaterial, Noise, Denoising, Filters, Quality

\section{Introduction}

Image denoising can be considered as a component of processing or as a process itself. Image denoising involves the manipulation of the image data to produce a visually high quality image. Images get often corrupted by additive and multiplicative noise. In today's real time applications and requirements resolution we get from normal images is not sufficient. We need look insight its crystallographic structure, topography, morphology etc of a substance. As nanoscopic image has got wide and significant use in the medical research and applications and in many other domains. Due to acquisition TEM images contain electronic noise and white diffraction artifacts localized on the edges of the Nanomaterials Various types of filters have been proposed for removal of noise in these microscopic images. This paper discusses the major types of noise used in simulation in the first part, few types of filters being simulated on a nanoscopic image in the second part and comparative analysis in the third part.

\section{Noise In An Microscopic Image}

We define noise as an unwanted component of the image. Noise occurs in images for many reasons. Image is the sum of the true pixel value and a random Gaussian distributed noise value. As the name indicates, this type of noise has a Gaussian distribution, which has a bell shaped probability distribution function.

Poisson noise, is a basic form of uncertainty associated with the measurement of light, inherent to the quantized nature of light and the independence of photon detections. Its expected magnitude is signal-dependent and constitutes the dominant source of image noise except in low-light conditions. The magnitude of poisson noise varies across the image, as it depends on the image intensity. This makes removing such noise very difficult.

Salt and pepper noise is an impulse type of noise, which is also referred to as intensity spikes. This is caused generally due to errors in data transmission. It has only two possible values, $a$ and $b$. The probability of each is typically less than 0.1 . The corrupted pixels are set alternatively to the minimum or to the maximum value, giving the image a "salt and pepper" like appearance. [2]

Speckle noise is a multiplicative noise. It is signal dependent, non-Gaussian \& spatially dependent. Due to microscopic variations in the surface, roughness within one pixel, the received signal is subjected to random variations in phase and amplitude. The variations in phase which are added constructively results in strong intensities while other which are added destructively results in low intensities. This variation is called as Speckle.[1] 


\section{A. Bilateral Filter}

\section{Denoising Filters}

Bilateral filtering is a non-linear filtering technique. It extends the concept of Gaussian smoothing by weighting the filter coefficients with their corresponding relative pixel intensities. Pixels that are very different in intensity from the central pixel are weighted less even though they may be in close proximity to the central pixel. This is effectively a convolution with a non-linear Gaussian filter, with weights based on pixel intensities. This is applied as two Gaussian filters at a localized pixel neighbourhood, one in the spatial domain, named the domain filter, and one in the intensity domain, named the range filter. Bilateral filter compares the intensity of the pixel to be filtered with the surrounding filtered intensities instead of the noisy ones. [3].

$$
I(\mathbf{x})=\frac{1}{C} \sum_{y \in N(x)} e^{\frac{-\| \mathbf{y}-\left.\mathbf{x}\right|^{2}}{2 \sigma_{d}^{2}}} e^{\frac{-|f(\mathbf{y})-I(\mathbf{x})|^{2}}{2 \sigma_{r}^{2}}} I(y)
$$

Fig.1 Bilateral Filter Equation

\section{B. Weiner Filter using DWT}

Wiener filter minimizes the mean square error between the uncorrupted signal and the estimated signal. Discrete Wavelet Transform analyzes the signal by successive use of low pass and high pass filtering to decompose the signal into its coarse and detail information. This denoising algorithm de-noise image using Wiener filter for Low frequency domain and using soft thresholding for de-noise High-frequencies domains. This approach is gives better results than (DWT or Wiener) de-noising. [4,8]

\section{Hybrid Median Filter}

Median filter is widely used in digital image processing for removing noise in digital images. Although it does not shift edges, the median filter does remove fine lines and detail, and round corners. A more advanced version of this filter, which avoids these problems, is the hybrid median. Hybrid median filtering preserves edges better than a $\mathrm{NxN}$ square kernel-based median filter because data from different spatial directions are ranked separately. Three median values are calculated in the $\mathrm{NxN}$ box: MR is the median of horizontal and vertical $\mathrm{R}$ pixels, and $\mathrm{MD}$ is the median of diagonal $\mathrm{D}$ pixels. The filtered value is the median of the two median values and the central pixel C: median ([MR, MD, C]). [5]

$$
\left[\begin{array}{ccccc}
D & * & R & * & D \\
* & D & R & D & * \\
R & R & D C R & R & R \\
* & D & R & D & * \\
D & * & R & * & D
\end{array}\right]
$$

Fig. 3 Formulation of Filtered Value

\section{Dual Vectorial ROF Filter}

Regularity is of central importance in computer vision. Total variation preserves edges and does not requires any prior information about the blurred image computed. One approach is to replace norm $l 2$ in Tikhonov Regularization with the norm $l 1$, i.e., the 1-norm of the first spatial derivation of the solution. This is called the total variation (TV) regularization. This method will help to obtain the discontinuities or steep gradients in the restored image. This procedure minimizes the vectorial total variation norm.[6] Let us consider a vectorial (or M-dimensional or multichannel) function $u$, such as a color image or a vector field, defined on a bounded open domain $\Omega \subset \mathrm{R}^{\mathrm{N}}$ as $\mathrm{x} \rightarrow \mathrm{u}(\mathrm{x}):=\left(\mathrm{u} 1(\mathrm{x}), \ldots, \mathrm{u}_{\mathrm{M}}(\mathrm{x})\right), \mathrm{u}: \rightarrow \mathrm{R}^{\mathrm{M}}$,

$$
\inf _{\mathrm{u}} \sup _{\mid \mathrm{p} \leq 1}\left\{\langle\mathrm{u}, \nabla \cdot \mathrm{p}\rangle_{L^{2}\left(\Omega, \mathbb{R}^{M}\right)}+\frac{1}{2 \lambda}\|\mathrm{f}-\mathrm{u}\|_{L^{2}\left(\Omega ; \mathbb{R}^{M}\right)}^{2}\right\}
$$

Fig. 2 Formulation of Vectorial TV Norm

Which is convex in $u$ and concave in $p$ and the set $\{|p|<=1\}$ is bounded and convex.[11,12]

E. Fuzzy Histogram Equalization

It proposes a novel modification of the brightness preserving dynamic histogram equalization technique to improve its brightness preserving and contrast enhancement abilities while reducing its 
computational complexity. This technique, called uses fuzzy statistics of digital images for their representation and processing. Representation and processing of images in the fuzzy domain enables the technique to handle the inexactness of gray level values in a better way, resulting in improved performance. Besides, the imprecision in gray levels is handled well by fuzzy statistics, fuzzy histogram, when computed with appropriate fuzzy membership function, does not have random fluctuations or missing intensity levels and is essentially smooth. This helps in obtaining its meaningful partitioning required for brightness preserving equalization.[7]

\section{Simulation Results}

The four types of noise are added to the original image ranging from $1 \%$ to $9 \%$ and are filtered with the above mentioned filters. The filtered image is compared with the original image on the basis of four characteristics shown in the Fig. 4.1 to 4.16

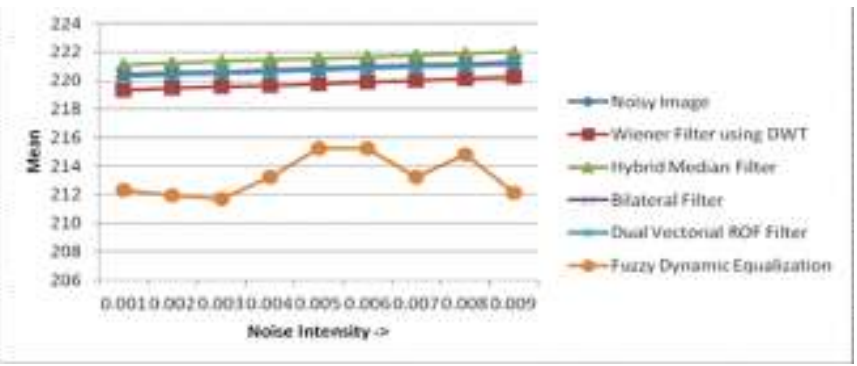

Fig. 4.1 Mean of Image after applying various filters on Gaussian Noise corrupted image.

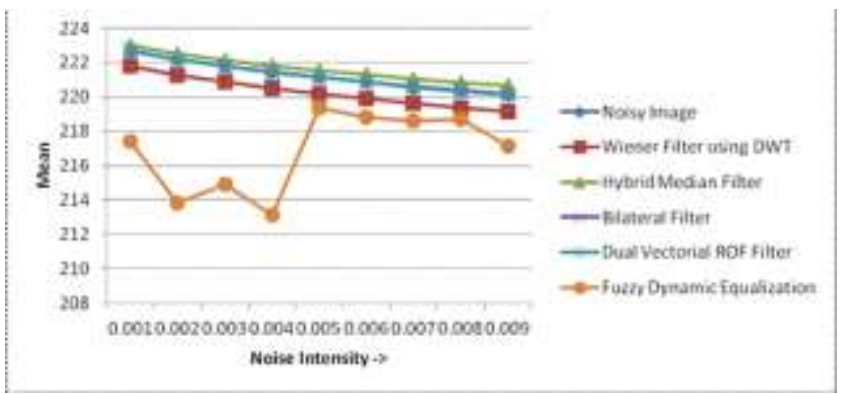

Fig.4.2 Mean of Image after applying various filters on Speckle Noise corrupted image

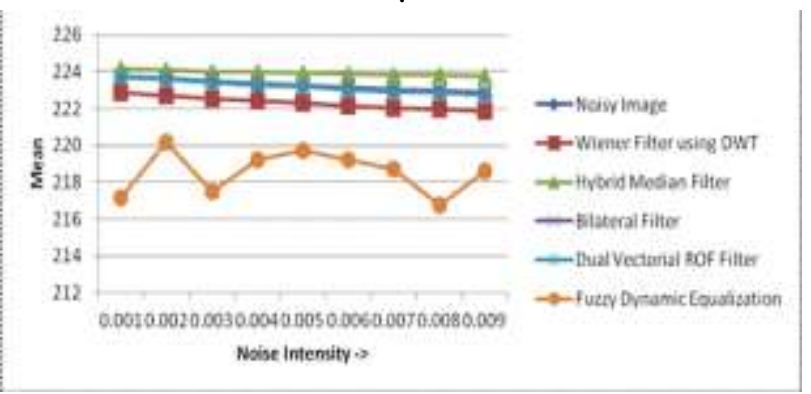

Fig.4.3 Mean of Image after applying various filters on Salt \& Pepper Noise corrupted image.

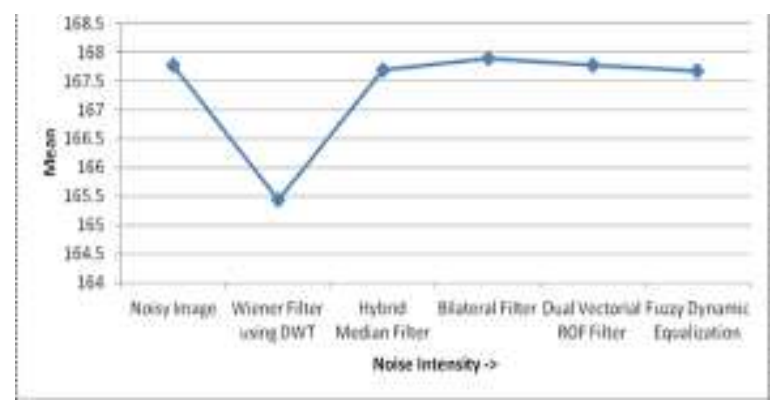

Fig.4.4 Mean of Image after applying various filters on Salt \& Pepper Noise corrupted image. 


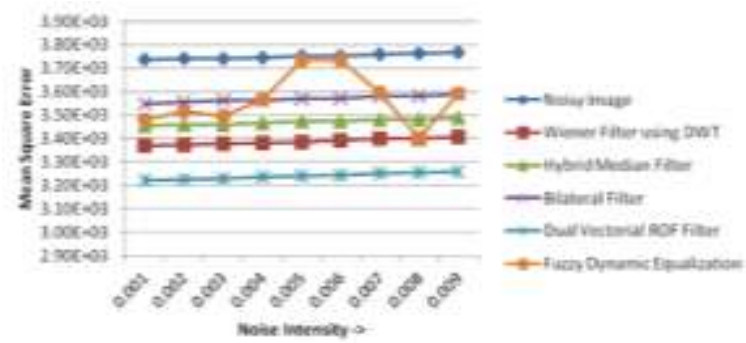

Fig.4.5 Mean Square Error of Image after applying various filters on Gaussian Noise corrupted image.

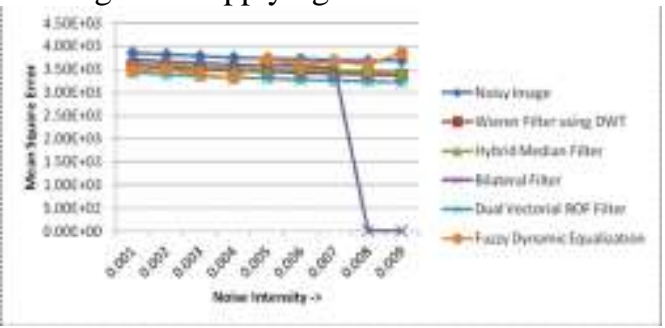

Fig.4.6 Mean Square Error of Image after applying various filters on Speckle Noise corrupted image.

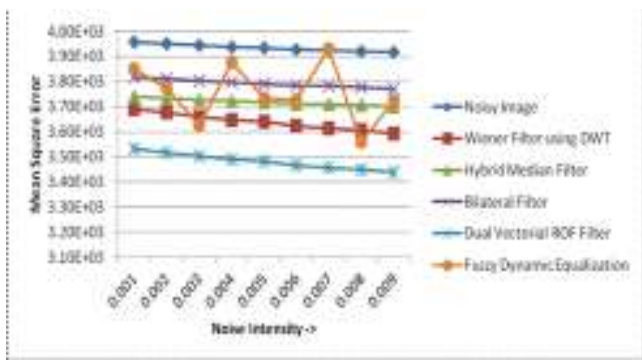

Fig.4.7 Mean Square Error of Image after applying various filters on Salt \& Pepper Noise corrupted image.

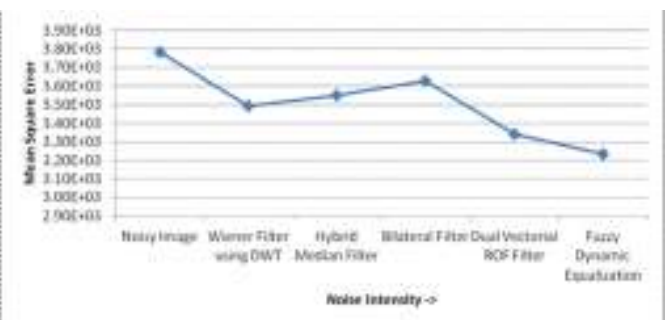

Fig.4.8 Mean Square Error of Image after applying various filters on Poisson Noise corrupted image.

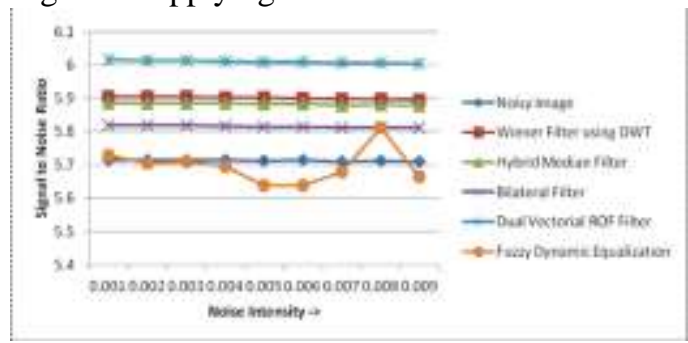

Fig.4.9 Signal to Noise Ratio of Image after applying various filters on Gaussian Noise corrupted image.

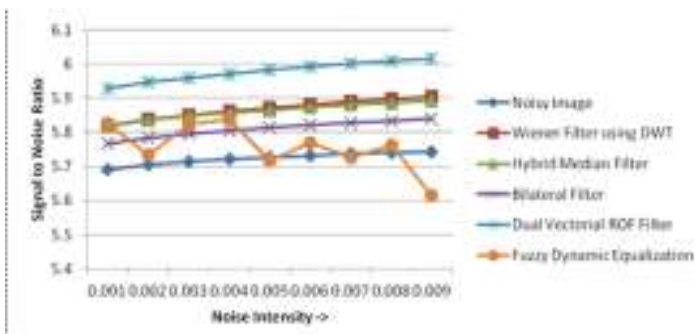

Fig.4.10 Signal to Noise Ratio of Image after applying various filters on Speckle Noise corrupted image. 


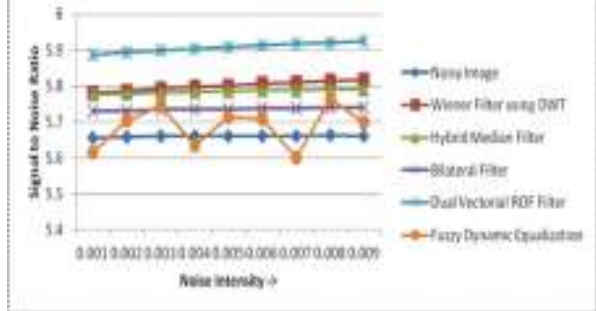

Fig.4.11 Signal to Noise Ratio of Image after applying various filters on Salt \& Pepper Noise corrupted image.

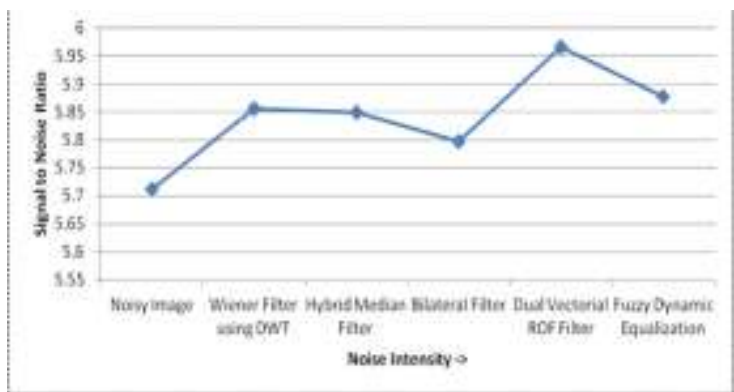

Fig.4.12 Signal to Noise Ratio of Image after applying various filters on Poisson Noise corrupted image.

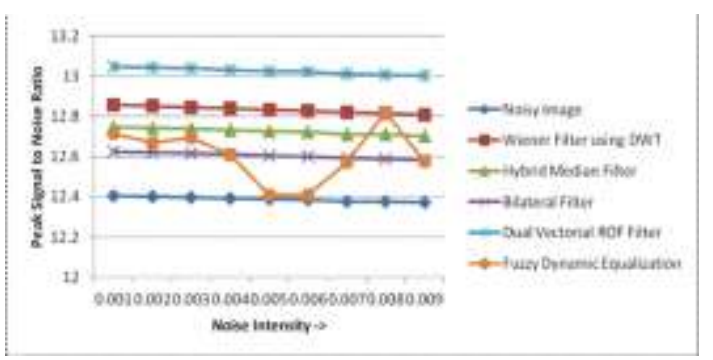

Fig.4.13 Peak Signal to Noise Ratio of Image after applying various filters on Gaussian Noise corrupted image.

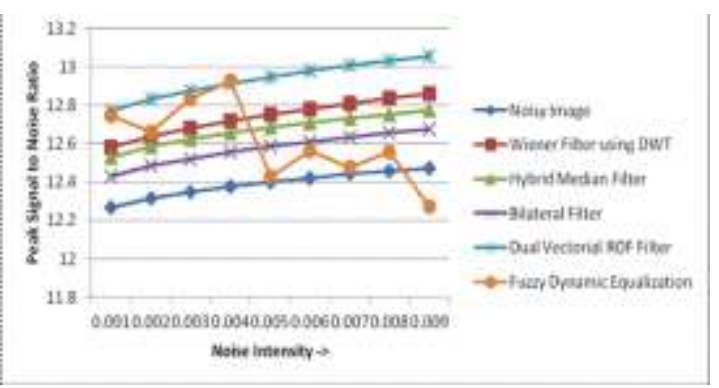

Fig.4.14 Peak Signal to Noise Ratio of Image after applying various filters on Speckle Noise corrupted image.

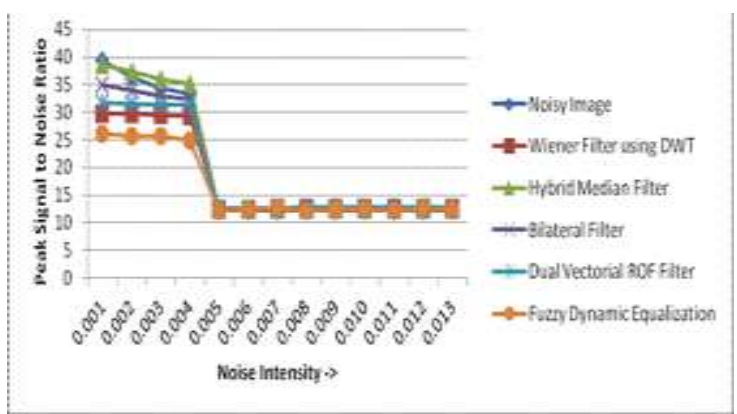

Fig.4.15 Peak Signal to Noise Ratio of Image after applying various filters on Salt \& Pepper Noise corrupted image. 


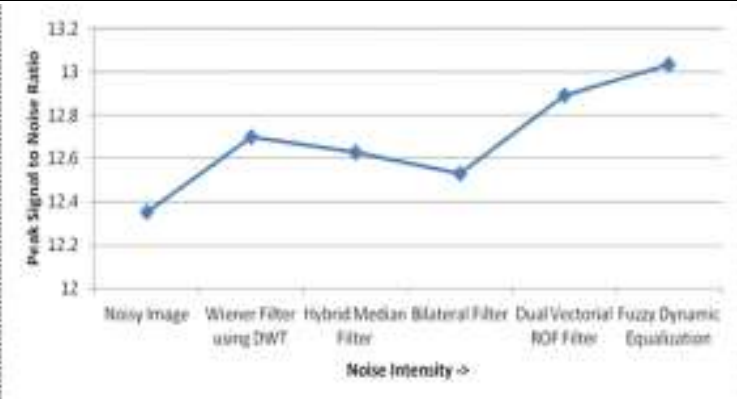

Fig.4.16 Peak Signal to Noise Ratio of Image after applying various filters on Poisson Noise corrupted image.

TABLE 5.1

AVERAGE MEAN VALUES OF FILTERED IMAGES

\begin{tabular}{|l|c|c|c|c|}
\hline MEAN RESULTS & Gaussian Noise & $\begin{array}{l}\text { Speckle } \\
\text { Noise }\end{array}$ & $\begin{array}{l}\text { Salt \& Pepper } \\
\text { Noise }\end{array}$ & $\begin{array}{l}\text { Poisson } \\
\text { Noise }\end{array}$ \\
\hline Noisy & 220.7368 & 221.2334 & 223.2045 & 221.621 \\
\hline Wiener Filter using DWT & $\mathbf{2 1 9 . 7 8 4 8}$ & 220.2959 & 222.3188 & 220.6939 \\
\hline Hybrid Median Filter & 221.5626 & 221.6444 & 223.9298 & 222.0618 \\
\hline Bilateral Filter & 220.8592 & 221.2602 & 223.2843 & 221.6503 \\
\hline Dual Vectorial ROF Filter & 220.7411 & 221.2407 & 223.2132 & 221.6225 \\
\hline Fuzzy Dynamic Equalization & 213.3209 & $\mathbf{2 1 6 . 8 7 6 5}$ & $\mathbf{2 1 8 . 5 5 8 7}$ & $\mathbf{2 1 2 . 6 3 4 4}$ \\
\hline
\end{tabular}

TABLE 5.2

AVERAGE MEAN SQUARE ERROR VALUES OF THE FILTERED IMAGES

\begin{tabular}{|l|c|c|c|c|}
\hline $\begin{array}{l}\text { MEAN SQUARE ERROR } \\
\text { RESULTS }\end{array}$ & Gaussian Noise & $\begin{array}{l}\text { Speckle } \\
\text { Noise }\end{array}$ & $\begin{array}{l}\text { Salt \& Pepper } \\
\text { Noise }\end{array}$ & $\begin{array}{l}\text { Poisson } \\
\text { Noise }\end{array}$ \\
\hline Noisy & 3751.6888 & 3752.044 & 3935.5444 & 3785 \\
\hline Wiener Filter using DWT & 3387.1556 & 3461.322 & 3638.4333 & 3494.3 \\
\hline Hybrid Median Filter & 3471.3111 & 3516 & 3719.0556 & 3549.8 \\
\hline Bilateral Filter & 3569.8725 & $\mathbf{2 8 1 5 . 5 9 3}$ & 3793.125 & 3628.395 \\
\hline Dual Vectorial ROF Filter & $\mathbf{3 2 4 0 . 2 3 3 3}$ & 3309.378 & $\mathbf{3 4 8 2}$ & 3341.1 \\
\hline Fuzzy Dynamic Equalization & 3568.9444 & 3570.233 & 3756.7667 & $\mathbf{3 2 3 4}$ \\
\hline
\end{tabular}

TABLE 5.3

AVERAGE SIGNAL TO NOISE RATIO VALUES OF FILTERED IMAGE

\begin{tabular}{|l|l|l|l|l|}
\hline $\begin{array}{l}\text { SIGNAL TO NOISE RATIO } \\
\text { RESULTS }\end{array}$ & $\begin{array}{l}\text { Gaussian } \\
\text { Noise }\end{array}$ & $\begin{array}{l}\text { Speckle } \\
\text { Noise }\end{array}$ & $\begin{array}{l}\text { Salt \& Pepper } \\
\text { Noise }\end{array}$ & $\begin{array}{l}\text { Poisson } \\
\text { Noise }\end{array}$ \\
\hline Noisy & 5.7130 & 5.7238 & 5.6606 & 5.7126 \\
\hline Wiener Filter using DWT & 5.9004 & 5.8682 & 5.8015 & 5.8555 \\
\hline Hybrid Median Filter & 5.8827 & 5.8612 & 5.7853 & 5.8485 \\
\hline Bilateral Filter & 5.8154 & 5.8096 & 5.7362 & 5.7966 \\
\hline Dual Vectorial ROF Filter & $\mathbf{6 . 0 0 9 9}$ & $\mathbf{5 . 9 7 8 4}$ & $\mathbf{5 . 9 0 8 3}$ & $\mathbf{5 . 9 6 5 1}$ \\
\hline Fuzzy Dynamic Equalization & 5.6967 & 5.7566 & 5.6875 & 5.8774 \\
\hline
\end{tabular}

TABLE 5.4

AVERAGE PEAK SIGNAL TO NOISE RATIO VALUES OF FILTERED IMAGES

\begin{tabular}{|l|l|l|l|l|}
\hline $\begin{array}{l}\text { PEAK SIGNAL TO NOISE } \\
\text { RATIO RESULTS }\end{array}$ & $\begin{array}{l}\text { Gaussian } \\
\text { Noise }\end{array}$ & $\begin{array}{l}\text { Speckle } \\
\text { Noise }\end{array}$ & $\begin{array}{l}\text { Salt \& Pepper } \\
\text { Noise }\end{array}$ & $\begin{array}{l}\text { Poisson } \\
\text { Noise }\end{array}$ \\
\hline Noisy & 12.3885 & 12.3886 & 12.1807 & 12.3502 \\
\hline Wiener Filter using DWT & 12.8324 & 12.7392 & 12.5218 & 12.6972 \\
\hline Hybrid Median Filter & 12.7258 & 12.671 & 12.4265 & 12.6287 \\
\hline Bilateral Filter & 12.6044 & 12.5720 & 12.3410 & 12.53 \\
\hline Dual Vectorial ROF Filter & $\mathbf{1 3 . 0 2 5 0}$ & $\mathbf{1 2 . 9 3 4 2}$ & $\mathbf{1 2 . 7 1 2 7}$ & $\mathbf{1 2 . 8 9 1 9}$ \\
\hline Fuzzy Dynamic Equalization & 12.6073 & 12.6082 & 12.3845 & 13.0335 \\
\hline
\end{tabular}




\section{Conclusion}

We used the greyscale TEM Image adding four types of noise (Speckle, Gaussian, Poisson and Salt \& Pepper) in original image and de-noised all noisy images by all filters. Graphs are shown in Fig 4.1 to 4.16 on the basis of four parameters (Mean, Mean Square Error, Signal to Noise Ratio \& Peak Signal to Noise Ratio). The result for in each category for every filter is summarized in tables 4.1 to 4.4. The following table concludes the complete discussion made in paper.

TABLE 6.1

AN APPROPRIATE FILTER AGAINST EACH TYPE OF NOISE AND CHARACTERISTIC PAIR

\begin{tabular}{|l|l|l|l|l|}
\hline & Gaussian Noise & Speckle Noise & Salt \& Pepper Noise & Poisson Noise \\
\hline Mean & $\begin{array}{l}\text { Wiener Filter using } \\
\text { DWT }\end{array}$ & $\begin{array}{l}\text { Fuzzy Dynamic } \\
\text { Equalization }\end{array}$ & $\begin{array}{l}\text { Fuzzy Dynamic } \\
\text { Equalization }\end{array}$ & $\begin{array}{l}\text { Fuzzy Dynamic } \\
\text { Equalization }\end{array}$ \\
\hline $\begin{array}{l}\text { Mean Square } \\
\text { Error }\end{array}$ & $\begin{array}{l}\text { Dual Vectorial ROF } \\
\text { Filter }\end{array}$ & Bilateral Filter & $\begin{array}{l}\text { Dual Vectorial ROF } \\
\text { Filter }\end{array}$ & $\begin{array}{l}\text { Fuzzy Dynamic } \\
\text { Equalization }\end{array}$ \\
\hline $\begin{array}{l}\text { Signal to Noise } \\
\text { Ratio }\end{array}$ & $\begin{array}{l}\text { Dual Vectorial ROF } \\
\text { Filter }\end{array}$ & $\begin{array}{l}\text { Dual Vectorial ROF } \\
\text { Filter }\end{array}$ & $\begin{array}{l}\text { Dual Vectorial ROF } \\
\text { Filter }\end{array}$ & $\begin{array}{l}\text { Dual Vectorial } \\
\text { ROF Filter }\end{array}$ \\
\hline $\begin{array}{l}\text { Peak Signal to } \\
\text { Noise Ratio }\end{array}$ & $\begin{array}{l}\text { Dual Vectorial ROF } \\
\text { Filter }\end{array}$ & $\begin{array}{l}\text { Dual Vectorial ROF } \\
\text { Filter }\end{array}$ & $\begin{array}{l}\text { Dual Vectorial ROF } \\
\text { Filter }\end{array}$ & $\begin{array}{l}\text { Dual Vectorial } \\
\text { ROF Filter }\end{array}$ \\
\hline
\end{tabular}

From simulation results and denoised images it is found that for each kind of noise Dual Vectorial ROF Filter works better than others.

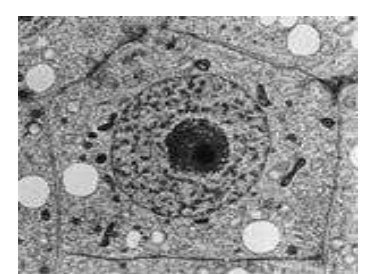

Fig. 6.1 Original Image

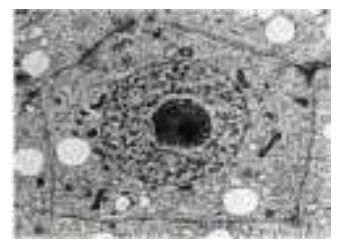

Fig. 6.2 Image with Gaussian Noise

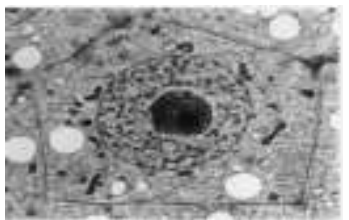

Fig. 6.3 Filtered Image of Fig.6.2 by Dual Vectorial ROF Filter.

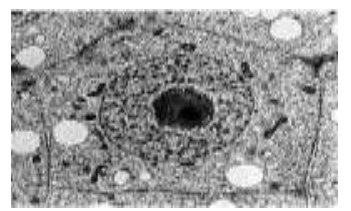

Fig. 6.4 Image with Speckle Noise 0.005

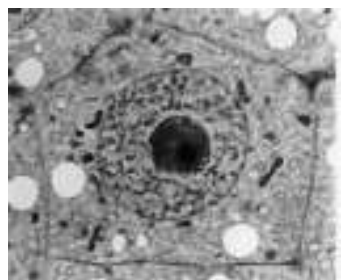

Fig. 6.5 Filtered Image of Fig.6.4 by Dual Vectorial ROF 


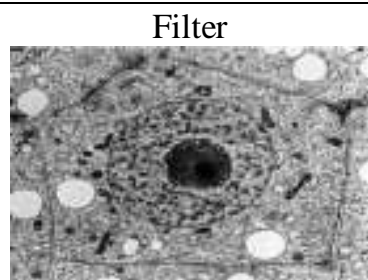

Fig. 6.6 Image with Salt \& Pepper Noise 0.005

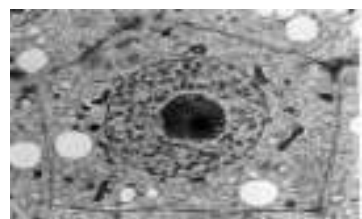

Fig. 6.7 Filtered Image of Fig.6.6 by Dual Vectorial ROF Filter.

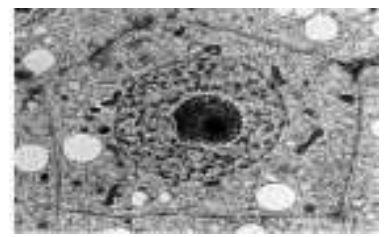

Fig. 6.8 Image with Poisson Noise 0.005

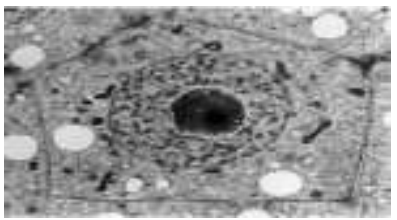

Fig. 6.9 Filtered Image of Fig.6.8 by Dual Vectorial ROF Filter.

\section{Future Scope}

Though Dual Vectorial ROF Filters retains the structure in the image but do not capture very fine details due to smoothing. Further these algorithms can be modified to overcome the drawbacks.

\section{References}

[1] Charles Boncelet (2005). "Image Noise Models". In Alan C. Bovik. Handbook of Image and Video Processing. Academic Press. ISBN 0-12-119792-1.

[2] Rafael C. Gonzalez, Richard E. Woods (2007). Digital Image Processing, Pearson Prentice Hall. ISBN 0-13-168728-X

[3] Arnaud DeDecker1, JohnA.Lee 2, MichelVerleysen A principled approach to image denoising with similarity kernels involving patches Elsevier Neurocomputing 73 (2010) 1199-1209

[4] Václav MATZ, Marcel KREIDL, Radislav ŠMID and Stanislav ŠTARMAN, Ultrasonic Signal De-noising Using Dual Filtering Algorithm, 17th World Conference on Nondestructive Testing, 25-28 Oct 2008, Shanghai, China

[5] R.Vanithamani 1, G.Umamaheswari2, M.Ezhilarasi Published in: Proceeding ICNVS'10 Proceedings of the 12th international conference on Networking, VLSI and signal processing Pages 166-171 World Scientific and Engineering Academy and Society (WSEAS) Stevens Point, Wisconsin, USA @2010 table of contents ISBN: 978-960-474-162-5

[6] Xavier Bresson, Tony Chan, Fast dual minimization of the vectorial total variation norm and applications to color image processing, Inverse Problems \& Imaging (2008) Volume: 2, Issue 4, Pages: 455-484, ISSN: 19308337, DOI: 10.3934/ipi.2008.2.455

[7] D. Sheet, H. Garud, A. Suveer, J. Chatterjee and M. Mahadevappa, "Brightness Preserving Dynamic Fuzzy Histogram Equalization", IEEE Trans., Consumer Electronics, vol. 56, no. 4, pp. 2475 - 2480, Nov. 20

[8] Pawan Patidar, Manoj Gupta, Sumit Srivastava and Ashok Kumar Nagawat. Article:Image De-noising by Various Filters for Different Noise. International Journal of Computer Applications 9(4):45-50, November 2010. Published By Foundation of ComputerScience 